\title{
Avaliação de prevalência de calcificação da artéria carótida em ra- diografias panorâmicas na população goiana
}

Sara Lia Gonçalves, Jéssica de Oliveira Luiz Damascenoㄹ, Fernando Fortes Picoli², Juliano Martins Bueno $^{3}$, Mayara Barbosa Viandelli Mundim-Picoli4.

${ }^{1}$ Acadêmica do curso de Odontologia do Centro Universitário de Anápolis (UniEVANGÉLICA). ${ }^{2}$ Mestre em Odontologia - Universidade

Federal de Goiás. ${ }^{3}$ Radiologista e diretor científico do Centro Integrado de Radiodontologia (C.I.R.O.), ${ }^{4}$ Doutora em Odontologia- Universidade Federal de Goiás e Professora da área de Diagnóstico - Centro Universitário de Anápolis (UniEVANGÉLICA).

\begin{abstract}
Resumo
Objetivo: Este trabalho teve por finalidade investigar a prevalência de imagens compatíveis com calcificação da artéria carótida (CAC) como achado incidental em radiografias panorâmicas numa amostra da população goiana. Metodologia: Foram analisadas radiografias panorâmicas digitais obtidas de pacientes com idade igual ou superior à 40 anos, de ambos os gêneros, encaminhados ao serviço de radiologia de clínica particular. Um examinador, com conhecimento em radiologia, investigou a presença de áreas radiopacas, na altura das vértebras C3 e C4, com angulação de 45 graus formada com o ângulo da mandíbula, sugestivas de CAC. Resultados: Foi encontrada uma prevalência de 6,1\% de CAC na população estudada. Foi observado um risco mais elevado de desenvolvimento de CAC em pacientes com idade superior à 55 anos $(O R=2,92)$. Houve diferença estatisticamente significante entre a presença de CAC e gênero $(p=0,004)$ sendo as mulheres 1,60 vezes mais acometidas que os homens quando o desfecho se fez presente. Conclusão: A radiografia panorâmica representa um método com potencial para sugerir a presença de CAC, devendo o cirurgião-dentista estar atento à esta possibilidade de diagnóstico, contribuindo para a prevenção de eventos cardiovasculares e vasculocerebrais.
\end{abstract}

Palavras-chave: Calcificação na carótida; Radiografia panorâmica; Diagnóstico.

\section{Introdução}

O cirurgião-dentista, atualmente, está inserido em um processo de transição da Odontologia, onde a prática curativa-restauradora tem perdido ênfase para a Odontologia de prevenção que busca conhecer e colaborar com o estado de saúde geral do paciente ${ }^{1}$. O profissional da odontologia passa a ter papel essencial na equipe de saúde, não somente por se dedicar ao cuidado com a cavidade bucal, mas, também, por ser responsável na identificação das relações presentes entre as alterações sistêmicas e o complexo bucomaxilofacial².

A radiografia panorâmica, exame mais solicitado pelos cirurgiões-dentistas, não só proporciona uma visão geral das estruturas da cavidade oral, mas, também, permite a identificação e análise de alterações que não estão ligadas de forma direta com a Odontologia, o que permite a ampliação da utilidade da radiografia panorâmica e reforça o papel do profissional da odontologia na equipe multiprofissional da saúde ${ }^{3,4}$.

A arterosclerose é uma doença inflamatória crônica de origem multifatorial que ocorre em resposta à agressão endotelial, acometendo principalmente a camada íntima de artérias de médio e grande calibres, sendo caracterizada pela formação de placas composta por lipídios e tecido fibroso no interior dos vasos sanguíneos ${ }^{5,6}$. Estas placas, denominadas ateroma, com o passar do tempo podem levar a obstrução parcial ou total do vaso sanguíneo, sendo a artéria carótida frequentemente acometida por este processo ${ }^{7}$.

A obstrução parcial ou total dos vasos sanguíneos aumentam as chances de o paciente sofrer infarto agudo do miocárdio ou acidente vascular cerebral ${ }^{8,9}$. Para tanto, a maneira principal de prevenção da arterosclerose é a detecção de sua predisposição precocemente, ou seja, percebendo calcificações na artéria carótida $(\mathrm{CAC})^{10}$.

A percepção das calcificações na artéria carótida (CAC) pode ser conseguida com precisão através da radiografia panorâmica. Quando isso ocorre, os achados radiográficos são considerados incidentais e caracterizam como ferramenta complementar na prevenção dos acidentes cardiovasculares e vasculocerebrais $^{11}$.

Sendo assim, o objetivo deste artigo é investigar a prevalência de imagens compatíveis com calcificação da artéria carótida como achados incidentais em radiografias panorâmicas numa população do Estado de Goiás.

\section{Material e métodos}

O presente estudo é de caráter observacional e teve sua aprovação no Comitê de Ética e Pesquisa sob número CAAE 51066715.0.0000.5078.

A amostra deste estudo foi composta por radiografias panorâmicas digitais obtidas de pacientes com idade igual ou superior à 40 anos, de ambos os gêneros, encaminhados ao serviço de radiologia de clínica particular especializada do Estado de Goiás, com finalidades distintas, no período de janeiro à dezembro de 2014 e 2015.

As radiografias panorâmicas digitais que compuseram a amostra foram obtidas através do aparelho CRANEX ${ }^{\circledR}$ D sistema digital direto (Orion Corp.,Soredex, Helsinki, Finlândia) e adquiridas por um gerador de 
alta frequência $(40 \mathrm{kHz})$, sensibilizadas em um sensor CCD de 147.5 x $6.1 \mathrm{~mm}, 96 \mu \mathrm{m}$ de pixel, com tensão de tubo 57 - 85 kV e 10 mA de corrente, com 17,6 segundos de tempo de exposição. As imagens foram obtidas por um único profissional, treinado e com experiência na utilização do equipamento.

As imagens da amostra foram analisadas por um único examinador, com experiência em radiologia, utilizando o próprio programa do aparelho de raio-x panorâmico (DIGORA ${ }^{\circledR}$ for Windows 2.7 software, Orion Corp., Soredex, Helsinki, Finlândia), em um computador com sistema operacional Microsoft Windows XP Professional SP-2 (Microsoft Corp., Redmond, WA, EUA), com processador Intel $\mathbb{R}$ Core $^{\mathrm{TM}} 2$ Duo 1,86Ghz-6300 (Intel Corporation, EUA), placa de vídeo NVIDIA GeForce 6200 turbo cache (NVIDIA Corporation, EUA) e monitor EIZO - S2000 FlexScan, resolução de 1600×1200 pixels (EIZO Nanao Corporation Hakusan, Japão). O examinador investigou a presença de áreas radiopacas, na altura das vértebras C3 e C4, com angulação de 45 graus formada com o ângulo da mandíbula, sugestivas de calcificação da artéria carótida (Fig. 1-3).

\section{Resultados}

A amostra foi constituída de 3021 radiografias panorâmicas, sendo que foi encontrada uma prevalência de 6,1\% de CAC na população estudada (Tabela 1 e Fig 1-3). Observou-se um risco mais elevado de desenvolvimento de CAC em pacientes com idade superior à 55 anos $(\mathrm{OR}=2,92)$ (Tabela 2). Quanto à localização da calcificação, o lado direito foi mais acometido (Tabela 3).

Houve diferença estatisticamente significante entre a presença de CAC e gênero $(p=0,004)$ sendo as mulheres 1,60 vezes mais acometidas que os homens quando o desfecho se fez presente (Tabela 4).

\section{Discussão}

De acordo com Manzi et al., (2001)12 o processo de formação de placas ateroscleróticas tem início por meio de lesões às células do endotélio. Estas placas podem se tornar incrustadas com sais de cálcio e, nestas situações, são intituladas como ateromas. Ciclos de deterioração e reparação acontecem, repetidamente, e

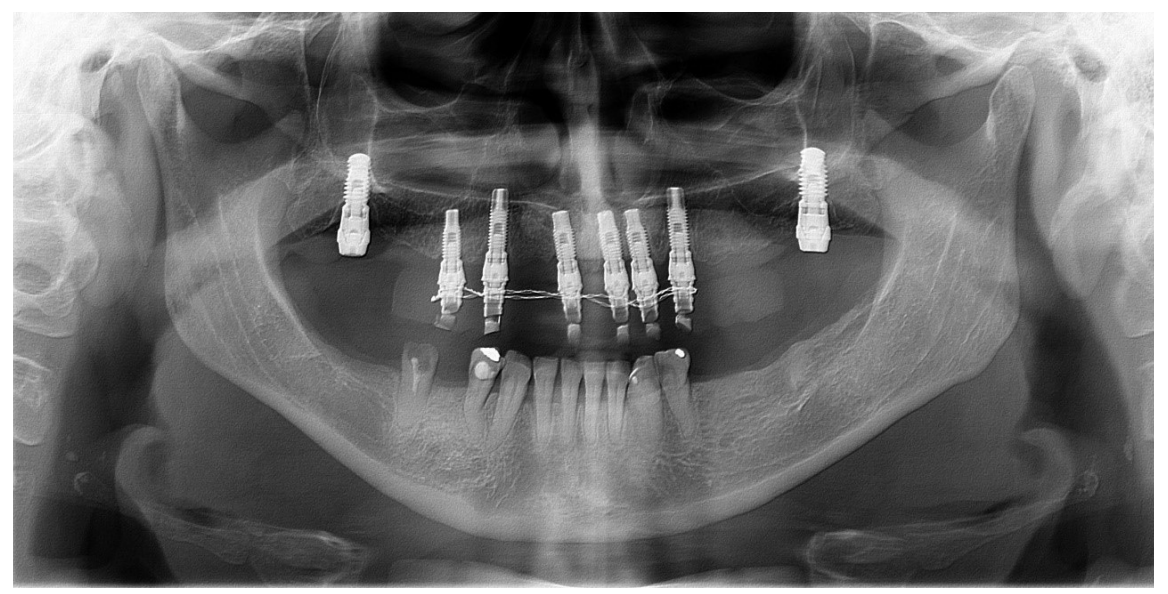

Figura 1 - Radiografia panorâmica evidenciando a presença de focos calcificados na região das vértebras $\mathrm{C} 3$ e C4 do lado direito e esquerdo do paciente.

Para a análise das imagens foi selecionado um ambiente favorável para a interpretação e as mesmas foram disposta em modo tela cheia sendo classificadas em: excluídas (imagens que não preenchiam aos critérios de inclusão deste estudo), CAC (radiografias incluídas e que apresentavam a imagem sugestiva de CAC, sendo então subdividas de acordo com a localização da calcificação em: unilateral direito, unilateral esquerdo e bilateral) e nenhuma evidência de CAC. Os dados foram tabulados em planilhas utilizando o programa Microsoft Office Excel para Windows (Microsoft Corporation, Washington, EUA). O software estatístico IBM-SPSS versão 19.0 foi utilizado para a realização de estatística descritiva. Foi utilizado o teste do qui-quadrado e Odds ratio (OR) para comparações entre as possíveis diferentes frequências de calcificação da artéria carótida entre diferentes gêneros e faixa etária. estas placas passam a sofrer hemorragia internamente e, posteriormente, ulcerações através do endotélio. Este processo promove a exposição das fibras colágenas que, por consequência, resulta no desenvolvimento de um trombo mural.

A embolização do trombo promove a obliteração das artérias cranianas, o que resulta em um acidente vascular cerebral (AVC) ou aumenta seu calibre até provocar isquemia cerebral pela diminuição do suprimento sanguíneo ${ }^{12-15}$.

As artérias mais frequentemente afetadas são a aorta e aquelas diretamente relacionadas, principalmente, a artéria carótida sendo que a deposição das placas de ateroma na artéria carótida culminam na redução do volume sanguíneo e oxigênio que irrigam os órgãos, sendo assim considerado como um dos principais fatores de risco associado ao desenvolvimento de acidente vascular cerebral e infarto agudo do miocár- 


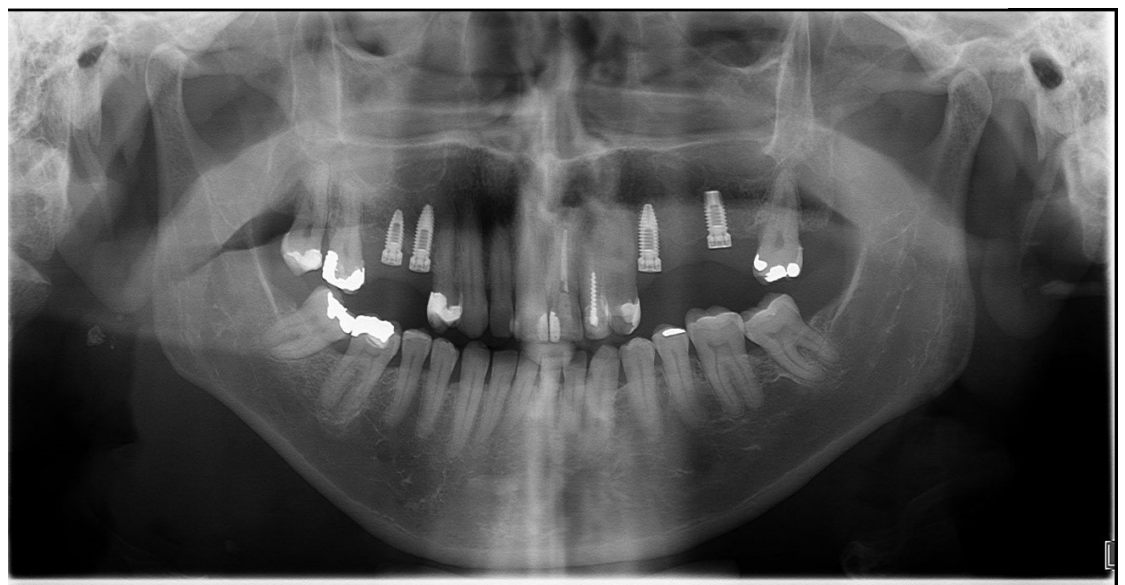

Figura 2 - Radiografia panorâmica evidenciando a presença de focos calcificados na região das vértebras C3 e C4 do lado direito do paciente.

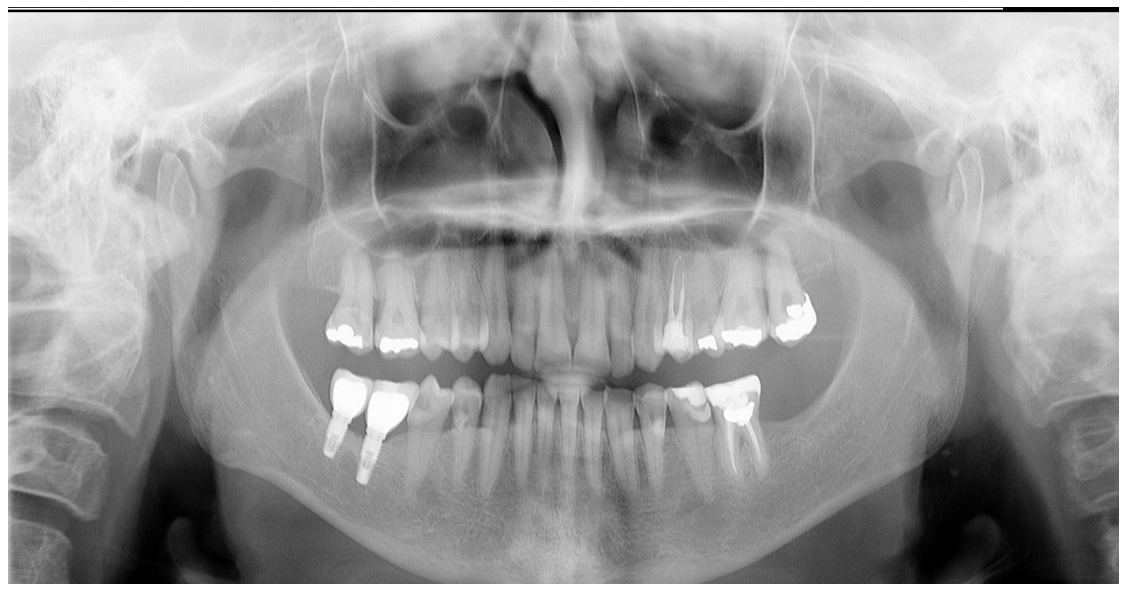

Figura 3 - Radiografia panorâmica evidenciando a presença de focos calcificados na região das vértebras C3 e C4 do lado esquerdo do paciente.

$\operatorname{dio}^{8,9,11,16}$.

Um evento coronário agudo é a primeira manifestação da doença aterosclerótica em pelo menos metade dos indivíduos que apresentam essa complicação ${ }^{10}$. O estudo de Moshfeghi ${ }^{9}$ evidencia a relação entre a CAC com o desenvolvimento de hipertensão arterial e infarto do miocárdio. No estudo desenvolvido por Lee ${ }^{17}$, os indivíduos que onde a presença de CAC foi evidenciada apresentaram taxas mais elevadas de doença arterial periférica. Desta forma, a identificação dos indivíduos assintomáticos que estão mais predispostos é crucial para a prevenção efetiva com a correta definição das metas terapêuticas individuais ${ }^{10}$.

Ateromas que se apresentam com tempo de evolução maior podem tornar-se com aspecto calcificado e serem visualizados através de radiografias panorâmicas ${ }^{3,18}$. As placas ateromatosas, quando estão calcificadas, podem ser vistas nas laterais das radiografias panorâmicas, na altura das vértebras $\mathrm{C} 3$ e C4, com angulação de 45 graus formada com o ângulo da mandíbula ${ }^{18-20}$

O primeiro estudo em que se indicou a radiografia panorâmica como método auxiliar para diagnóstico da CAC e risco de AVC foi composto pela análise de 1000 radiografias panorâmicas de homens entre 50 e 75 anos. Os autores identificaram calcificações na região da bifurcação da artéria carótida em $2 \%$ dos casos, sendo que dessas imagens $88 \%$ eram calcificações verdadeiras e $12 \%$ eram linfonodos calcificados ou cálculos salivares ${ }^{20}$.

Friedlander et al. (2005) ${ }^{16}$ observaram em 1548 panorâmicas, 4,2\% de ateromas de carótida em pacientes com mais de 50 anos. Faixa etária semelhante também observada no presente estudo sendo que $69,78 \%$ das radiografias panorâmicas que possuíam CAC eram de pacientes acima de 55 anos.

Taxas variáveis de prevalência de CAC foram observadas em diversos estudos em populações americanas e europeias. No estudo de Friedlander ${ }^{21}$ foi observada prevalência de $3 \%$, para Sisman22 esta taxa foi de 5,06\%, enquanto Brand ${ }^{23}$ encontrou valores de $9,4 \%$.

Numa revisão realizada por $\mathrm{Almog}^{3}$, foi encontrada uma prevalência de $3 \%$ a $5 \%$ de calcificação da artéria carótida na população geral, sendo percentagens mais elevadas evidenciadas em pacientes com doenças clínicas associadas com aterosclerose avançada. Este estudo realizado em uma população goiana 
Tabela 1 - Prevalência de calcificação da artéria carótida nas radiografias panorâmicas analisadas $(\mathrm{n}=3021)$

\begin{tabular}{|c|c|}
\hline Calcificações & Artéria Carótida \\
\hline Calcificada & $182(6,1 \%)$ \\
\hline Não-calcificada & $2839(93,9 \%)$ \\
\hline Total & $3021(100 \%)$ \\
\hline
\end{tabular}

Tabela 3 - Localização da calcificação da artéria carótida nas radiografias panorâmicas analisadas que apresentavam esta condição $(n=182)$.

\begin{tabular}{|c|c|}
\hline Localização da Calcificação & A. Carótida \\
\hline Calcificação Bilateral & $51(28,02 \%)$ \\
\hline $\begin{array}{c}\text { Calcificação unilateral direita } \\
\text { Calcificação unilateral } \\
\text { esquerda }\end{array}$ & $90(49,45 \%)$ \\
\hline Total & $41(22,53 \%)$ \\
\hline
\end{tabular}

observou a porcentagem de 6,1\%.

Apesar do consenso existente na utilidade da radiografia panorâmica para visualização da CAC em pacientes assintomáticos ${ }^{3,18}$, para Madden et al. $(2007)^{24}$ concluíram que este exame, quando comparado à ultrassonografia, apresenta baixa sensibilidade em detectar calcificação da artéria carótida.

As radiografias panorâmicas, apesar de fornecerem vi são sugestiva de CAC, elas não identificam o grau de obstrução da carótida e nem a precisa localização do ateroma. Sendo assim, outros recursos são necessários para fechamento de um diagnóstico definitivo. A ultrassonografia de Doppler é o exame mais indicado para a confirmação da presença, localização e tamanho dos ateromas de carótida. Trata-se de um exame de baixo custo quando comparado aos contrastes angiográficos e com a tomografia computadoriza$\mathrm{da}^{19}$.

Dessa forma, a minuciosa interpretação deste exame pelo cirurgião-dentista pode revelar achados incidentais, que não se relacionavam diretamente ao motivo principal para solicitação do mesmo, mas que podem contribuir para o restabelecimento da saúde do paciente, antecipando o tratamento do paciente e reduzindo os riscos de morbidade e mortalidade $7,23,26,27$.

\section{Conclusão}

A radiografia panorâmica representa um método com potencial para sugerir a presença de CAC na população goiana, devendo o cirurgião-dentista estar atento à esta possibilidade de diagnóstico, contribuindo para a prevenção de eventos cardiovasculares e vasculocerebrais.
Tabela 2 - Média de idade dos pacientes que apresentavam CAC em suas radiografias panorâmicas $(\mathrm{n}=182)$

\begin{tabular}{|c|c|}
\hline Idade & Calcificações \\
\hline Entre 40 e 54 anos & $55(30,22 \%)$ \\
\hline Acima de 55 anos & $127(69,78 \%)$ \\
\hline Total & $182(100 \%)$ \\
\hline
\end{tabular}

Tabela 4 - Prevalência de gênero dos pacientes que apresentavam CAC em suas radiografias panorâmicas $(\mathrm{n}=182)$.

\begin{tabular}{|c|c|}
\hline Gênero & Calcificações \\
\hline Masculino & $51(28,03 \%)$ \\
\hline Feminino & $131(71,97 \%)$ \\
\hline Total & $182(100 \%)$ \\
\hline
\end{tabular}

\section{Referências}

1.Romiti CBB. Análise da ocorrência de imagens sugestivas de calcificações da artéria carótida em radiografias panorâmicas. Mato Grosso do Sul. Dissertação [Mestrado em Saúde e Desenvolvimento] - Faculdade de Medicina da Universidade Federal de Mato Grosso do Sul; 2009.

2. Oppermann RV, Rosing CK. Periodontia - Ciência e Clínica. São Paulo: Artes Médicas. 2001, 458 p.

3. Almog DM, Illig KA, Carter LC, Friedlander AH, Brooks SL, Grimes RM. Diagnosis of non-dental conditions. Carotid artery calcifications on panoramic radiographs identify patients at risk for stroke. N Y State Dent J. 2004;70(8):20-5.

4. Nakamoto $T$, Taguchi $A$, Ohtsuka $M$, Suei $Y$, Fujita $M$, Tanimoto $K$, et al. Dental panoramic radiograph as a tool to detect postmenopausal women with low bone mineral 16 density: untrained general dental practitioners' diagnostic performance. Osteoporos Int. 2003;14(8):659-64.

5. Jayasooriya G, Thapar A, Shalhoub J, Davies AH. Silent cerebral events in asymptomatic carotid stenosis. J Vasc Surg. 2011;54(1):227-36.

6. Yun WS, Rho YN, Park UJ, Lee KB, Kim DI, Kim YW. Prevalence of asymptomatic critical carotid artery stenosis in Korean patients with chronic atherosclerotic lower extremity ischemia: is a screening carotid duplex ultrasonography worthwhile? J Korean Med Sci. 2010;25(8):1167-70.

7. de Weerd M, Greving JP, Hedblad B, Lorenz MW, Mathiesen $E B, O$ 'Leary $D H$, et al. Prevalence of asymptomatic carotid artery stenosis in the general population: an individual participant data meta-analysis. Stroke. 2010;41(6):1294-7.

8. Abecasis $\mathrm{P}$, Chimenos-Kustner E, Lopez-Lopez O. Orthopantomography contribution to prevent isquemic stroke. J Clin 
Exp Dent. 2014;6(2):e127-31.

9. Moshfeghi M, Taheri JB, Bahemmat N, Evazzadeh ME, Hadian $H$. Relationship between carotid artery calcification detected in dental panoramic images and hypertension and myocardial infarction. Iran J Radiol. 2014;11(3):e8714.

10. Xavier HT, Faria Neto JR, Assad MH, Rocha VZ, Sposito AC, Fonseca FA, et al. V Diretriz Brasileira de Dislipidemias e Prevenção da Aterosclerose. In: Cardiologia SBd, editor. Arquibos Brasileiros de Cardiologia. Rio de Janeiro2013. p. 30.

11. Cohen SN, Friedlander AH, Jolly DA, Date L. Carotid calcification on panoramic radiographs: an important marker for vascular risk. Oral Surg Oral Med Oral Pathol Oral Radiol Endod. 2002;94(4):510-4.

12. Manzi FR, Tuji FM, Almeida SM, Haiter Neto F, Boscolo FN. Radiografia panorâmica na identificação de pacientes com risco de AVC. Revista da APCD 2001; 55: 131 - 33.

13. Pontual MLA, Martins, MGBQ, Freire Filho, FWV, Haiter Neto F, Moraes M. Diagnóstico diferencial das calcificações da região cervical: revisão de literatura. Rev Assoc Paul Cir Dent 2003; 57: $429-433$.

14. Almong, BM, Tsimidis, K, Moss, ME, Gottlieb, RH, Carter, LC. Evualution of a training program for detection of carotid artery calcifications on panoramic radiographs. Oral Surg Oral Med Oral Pathol Oral Radiol Endod 2000; 90: 111-117.

15. Sung EC, Friedlander, AH, Kobashigawa, JA. The prevalence of calcified carotid atheromas on the panoramic radiographs of patients with dilated cardiomyopathy Oral Surg Oral Med Oral Pathol Oral Radiol Endod 2004; 97: 404- 407.

16. Friedlander $\mathrm{AH}$, Chang $\mathrm{TI}$, Aghazadehsanai N, Berenji GR, Harada ND, Garrett NR. Panoramic images of white and black post-menopausal females evidencing carotid calcifications are at high risk of comorbid osteopenia of the femoral neck. Dentomaxillofac Radiol. 2013;42(5):20120195.

17. Lee JS, Kim OS, Chung HJ, Kim YJ, Kweon SS, Lee YH, et al. The prevalence and correlation of carotid artery calcification on panoramic radiographs and peripheral arterial disease in a population from the Republic of Korea: the Dong-gu study. Dentomaxillofac Radiol. 2013;42(3):29725099.

18. Friedlander AH, Garret NR, Chin EE. Ultrasonographic confirmation of carotid artery atheromas diagnosed via panoramic radiography. J. Am. Dent. Assoc. 2005; 136: 635-40.

19. Eid NLM. Saúde bucal e aterosclerose da carótida. Revista Eletrô- nica de Jornalismo Científico. Disponível em: http:// www.comciencia. br/comciencia/handler.php?section=8\&edicao $=47 \&$ id $=586$ Acesso em: 12/11/2010

20. Friedlander $A H$, Lande $A$. Panoramic radiography identification of carotid arterial plaques. Oral Surg. Oral Med. Oral Pathol. Oral Radiol. Endod. 1981; 52 (1): 102-4.

21. Friedlander $A H$, Baker JD. Panoramic radiography: an aid in detecting patients at risk of cerebrovascular accident. J Am Dent Assoc. 1994;125(12):1598-603.
22. Sisman Y, Ertas ET, Gokce C, Menku A, Ulker M, Akgunlu F. The Prevalence of Carotid Artery Calcification on the Panoramic Radiographs in Cappadocia RegionPopulation. Eur J Dent. 2007;1(3):132-8.

23. Brand HS, Mekenkamp WC, Baart JA. [Prevalence of carotid artery calcification on panoramic radiographs]. Ned Tijdschr Tandheelkd. 2009;116(2):69-73.

24. Madden RP, Rindal DB, Ahmad M. Utility of panoramic radiographs in detecting cervical calcified carotid atheroma. Oral Surg. Oral Med. Oral Pathol. Oral Radiol. Endod. 2007; 103 (4): 543-8.

25. Mandian M, Tadinada A. Incidental findings in the neck region of dental implant patients: a comparison between panoramic radiography and CBCT. J Mass Dent Soc. 2014;63(2):425.

26. Frielander AH, Altman L. Carotid artery atheromas in postmenopausal women. J. Am. Dent. Assoc. 2001; 132 (8): 1130-6.

27. Glick M, Greenber BL. The potential role of dentists in identifying patient's risk of experiencing coronary heart disease events. J. Am. Dent. Assoc. 2005; 136 (11): 1541-6.

Recebido em : 21/10/2017

Aprovado em: 24/11/2017

Os autores declaram que não há conflitos de interesse.

\section{Autor correspondente:}

Mayara Barbosa Viandelli Mundim-Picoli Avenida Universitária qd. 60 It.08 Edifício Torre Macedônia 1 apto. 1102 Vila Santa Isabel Anápolis-Goiás Email: mayara.viandelli@gmail.com

Telefone: (62) 98222-1114 


\title{
Prevalence of carotid artery calcification in panoramic radiographs in goias state population
}

\begin{abstract}
Objective: This study aimed to investigate the prevalence of images compatible with carotid artery calcification (CAC) as an incidental finding on panoramic radiographs in a sample of Goias State population. Methodology: Digital panoramic radiographs were obtained from patients aged 40 years or older, of both genders, referred to a private radiology service. An examiner, with knowledge in radiology, investigated the presence of radiopaque areas, at the height of the C3 and C4 vertebrae, with an angulation of 45 degrees formed with the angle of the mandible, suggestive of CAC. Results: A prevalence of $6.1 \%$ of CAC was found in the studied population. A higher risk of developing CAC was observed in patients over 55 years of age $(O R=2.92)$. There was a statistically significant difference between the presence of CAC and gender ( $p=0.004)$, with women being 1.60 times more affected than men when the outcome was present. Conclusion: The panoramic radiograph represents a method with potential to suggest the presence of $\mathrm{CAC}$, and the dentist should be aware of this possibility of diagnosis, contributing to the prevention of cardiovascular and vasculocerebral events.
\end{abstract}

Keywords: Carotid calcification; Panoramic radiography; Diagnosis. 